TITLE:

\title{
New 3-bar prismatic tensegrity units
}

AUTHOR(S):

Liu, Heping; Zhang, Jingyao; Ohsaki, Makoto

CITATION:

Liu, Heping ... [et al]. New 3-bar prismatic tensegrity units. Composite Structures 2018, 184: 306-313

ISSUE DATE:

2018-01-15

URL:

http://hdl.handle.net/2433/243155

RIGHT:

(c) 2018. This manuscript version is made available under the CC-BY-NC-ND 4.0 license

http://creativecommons.org/licenses/by-nc-nd/4.0/; The full-text file will be made open to the public on 15 January 2020 in accordance with publisher's 'Terms and Conditions for Self-Archiving'; This is not the published version. Please cite only the published version; この論文は出版社版でありません。引用の際には出版社版をご確認ご利用ください。 


\title{
Submitted to Composite Structures
}

\section{New 3-bar prismatic tensegrity units}

\author{
Heping Liu ${ }^{\mathrm{a}, \mathrm{c} *}$, Jingyao Zhang ${ }^{\mathrm{b}}$ and Makoto Ohsaki ${ }^{\mathrm{a}}$ \\ a Department of Architecture and Architectural Engineering, Kyoto University, Kyoto-Daigaku Katsura, Nishikyo, \\ Kyoto 615-8540, Japan \\ b Department of Architecture and Urban Design, Nagoya City University, Chikusa-ku, Nagoya, 464-0083, Japan \\ ${ }^{\mathrm{c}}$ College of Mechanical and Electrical Engineering, Harbin Engineering University, Harbin 150001, China
}

*Corresponding author. E-mail address: liuheping1234@sohu.com

This study was carried out during the first author's stay at Kyoto University.

\begin{abstract}
Various methods have been presented for generating complex tensegrity structures by assembling simple units. Hence, generating new units will in turn benefit in obtaining various shapes of tensegrity structures. In this paper, we present three different new units by adding strings to the well-known 3-bar prismatic tensegrity unit. Analytical solutions are derived for their self-equilibrium analysis. Influences of structural parameters on internal forces of the members are investigated to study properties of the new tensegrity units.
\end{abstract}

Keywords: tensegrity unit; additional nodes and strings; self-equilibrium; prismatic unit.

\section{Introduction}

A tensegrity structure is composed of strings and bars [1]. In the strict definition of tensegrity structure, its rigid bars do not touch with each other, and are connected with strings [2]. All members of a tensegrity structure bear only axial forces [3]. Since a tensegrity structure with sparse members is statically indeterminate, self-equilibrium forces exist in the structure, it can bear large external forces and own light weight.

Tensegrity structures have many applications in different areas [4]. For example, when it is used for a planetary landing device, its continuous string net on the surface can support and protect bars inside it, and the net can absorb external shocks owing to its flexibility [5]. The above unique characteristics have made the tensegrity structures become a research hotspot.

Tensegrity units are the bases of some complex tensegrity structures. The needle tower built by Snelson in 1968 is a tensegrity structure consisting of axially connected several 3-bar tensegrity units [6]. The ball tensegrity robots by NASA are built with two 3-bar tensegrity units [7-9]. Caluwaerts and Carbajal [10] built a 12-bar tensegrity stacked by three 4-bar tensegrity units. Liu et al. [11] analyzed the tensegrity connecting units along its axis. Luo et $a l$. [12] constructed the spherical tensegrity structure with several tensegrity units. Therefore, developing new configurations of the tensegrity units will help obtain more various types of tensegrity structures.

Determination of self-equilibrium state is always a key point of research on shape design of tensegrity structures. In the self-equilibrium analysis, equilibrium matrix is formulated in various forms and investigated using various tools. Pellegrino [13] used singular value 
decomposition (SVD) of the equilibrium matrix to analyze a static, kinematic, or static/kinematic nature of any structural assembly. In his analysis, numbers of independent zero-energy deformation modes and independent states of self-stress were computed to classify the structures. Zhang et al. [14] decomposed the equilibrium matrix to compute the independent states of self-stress. An optimization method for the purpose of maximizing the global rigidity of the structure is applied to determine the combination coefficients of the independent symmetric self-stress states. Xu et al. [15] obtained the independent states of self-stresses and internal mechanisms of tensegirty structures by applying SVD to numerical expression of the equilibrium matrix. The method based on simulated annealing was adopted to further find a feasible state of self-stresses. Through comparison of dimension of the structure with rank deficiency of the equilibrium equations with respect to nodal coordinates, Zhang et al. [16] presented the necessary conditions for guaranteeing that a free-standing prestressed structure is non-degenerate.

Prestressed tensegrity structures must satisfy equilibrium equations defined by structural parameters, which conversely means that the equilibrium equations characterize the properties of structural parameters. Moreover analytical solutions, rather than numerical ones, of structural parameters can reflect relations between the parameters. Tibert and Pellegrino [17] solved equilibrium equations in symbolic form to obtain easily equilibrium conditions of the well-known prismatic tensegrity units.

In this paper, we present several new configurations for tensegrity units by adding strings to the existing 3-bar prismatic tensegrity units. Equilibrium equations are solved in symbolic form to obtain analytical solutions of structural parameters, aiming at finding general characteristics of the new tensegrity structures studied in this paper.

\section{Definition of prismatic tensegrity unit}

We consider a 3-bar prismatic tensegrity unit as shown in Fig.1(a). The thick lines in the figure are bars carrying compression and the thin lines refer to strings carrying tension. All members of the structure can be classified into four kinds: bars, declining strings, upper level strings, and lower level strings. Equilibrium of the tensegrity unit is dependent on $\alpha$ $\left(\alpha \in\left[-180^{\circ}, 180^{\circ}\right]\right)$, which is the angle projected on the lower plane and rotated from node 1 to node 4 as shown in Fig. 1(a). Note here that nodes 1 and 4 are connected by a bar.

When the 3-bar tensegrity unit is at self-equilibrium state, the angle $\alpha$ must satisfy [18]

$$
\alpha= \pm 150^{\circ}
$$

Two 3-bar tensegrity units corresponding to $\alpha=150^{\circ}$ and $-150^{\circ}$ are distinguished by the connectivity of declining strings. Figure 1(a) shows the case of $\alpha=150^{\circ}$, where nodes 1,2 and 3 are connected to nodes 6,4 and 5, respectively, by the declining strings. By contrast, when $\alpha=-150^{\circ}$, nodes 1,2 and 3 are connected to nodes 5, 6 and 4, respectively. Zhang et al. [19] showed that strings can be added between the existing nodes, as shown with dotted lines in Fig.1(b), to make the 3-bar tensegrity units at equilibrium with $\alpha$ in the range of $\left[-180^{\circ},-150^{\circ}\right] \cup\left[150^{\circ}, 180^{\circ}\right]$.

In this paper, we use the structure in Fig.1(a) as the base structure. A node is added to each upper level string, and the additional nodes are connected to the nodes on the lower 
plane by the additional strings.

\section{New configurations by addition of members}

To generate new units, every string on the upper plane of the 3-bar prismatic structure in Fig. 1(a) is split into two strings, called first and second upper level strings, by the additional node. Moreover, there are three different cases for connectivity of an additional string connecting the additional node to an existing node on the lower plane, as shown in Fig. 2. For each additional node, there are three strings connecting it to the existing nodes, and therefore, these three strings and the four nodes connected to the additional node have to be on the same plane to satisfy self-equilibrium. Accordingly, topology of the unit is classified into three types by the group of four nodes as listed in Table 1 , where $\{i, j, k, l\}(i, j, k, l \in\{1, \ldots, 9\})$ indicates the nodes $i, j, k$ and $l$ are on the same plane.

If there exists a structure at self-equilibrium for a specified value of $\alpha=\alpha^{*}$, it is easily seen from symmetry property that there exists another configuration corresponding to $\alpha=-\alpha^{*}$.

Therefore, we investigate mainly the structure with $\alpha \in\left[\begin{array}{ll}0^{\circ}, & 180^{\circ}\end{array}\right]$.

Let $r_{1}$ and $r_{2}$ denote radii of the circles containing the existing nodes respectively on the upper and lower planes as shown in Fig.1(b). Height of the 3-bar unit is denoted by $H$. Let $\boldsymbol{N}_{i}$ $(i=1, . ., 9)$ denote coordinate vector of the $i$ th node. Coordinates of the nodes located on the lower plane can be written as

$$
\boldsymbol{N}_{k}=\left[\begin{array}{c}
r_{1} \cos [2 \pi(k-1) / 3] \\
r_{1} \sin [2 \pi(k-1) / 3] \\
0
\end{array}\right] \quad(k=1,2,3)
$$

Nodal coordinates on the upper plane can be written as

$$
\boldsymbol{N}_{k}=\left[\begin{array}{c}
r_{2} \cos [2 \pi(k-4) / 3+\alpha] \\
r_{2} \sin [2 \pi(k-4) / 3+\alpha] \\
H
\end{array}\right] \quad(k=4,5,6)
$$

Since an additional node must be located inside the triangle enclosed by the existing nodes, coordinates of the additional nodes can be defined by coordinates of the existing nodes with the barycentric coordinates. Expressions for calculating coordinates of the additional nodes depend on connectivity of the additional strings. In Type 1, coordinates of the three additional nodes can be written as

$$
\begin{aligned}
& \boldsymbol{N}_{7}=c_{1} \boldsymbol{N}_{6}+c_{2} \boldsymbol{N}_{4}+\left(1-c_{1}-c_{2}\right) \boldsymbol{N}_{3} \\
& \boldsymbol{N}_{8}=c_{1} \boldsymbol{N}_{5}+c_{2} \boldsymbol{N}_{6}+\left(1-c_{1}-c_{2}\right) \boldsymbol{N}_{2} \\
& \boldsymbol{N}_{9}=c_{1} \boldsymbol{N}_{4}+c_{2} \boldsymbol{N}_{5}+\left(1-c_{1}-c_{2}\right) \boldsymbol{N}_{1}
\end{aligned}
$$

where $c_{1}, c_{2},\left(c_{1}+c_{2}\right) \in(0,1)$.

When $r_{1}, r_{2}, H, c_{1}, c_{2}$ and $\alpha$ are given, nodal coordinates can be calculated easily. However, arbitrary assignments of these parameters might not result in a self-equilibrated configuration. 


\section{Analytical Solutions}

In this section, we present the analytical conditions for self-equilibrium of the three new tensegrity units consisting of additional nodes and strings. Self-equilibrium of a tensegrity is decided by its nodal locations as well as the combination of internal forces in all the members. There are six kinds of members in the tensegrity units investigated in this paper. The members of the same kind are assumed to have the same length and internal forces according to symmetry; i.e., there are six different force densities in the structures. Since there are three types of nodes, which are the nodes on the lower plane, the nodes on the upper plane and the additional nodes, we can establish nine independent equilibrium equations, three equations for each type of nodes. Symbolic computation software package Maple 2016 [19] is used for assisting derivation of analytical solutions.

\subsection{Type 1}

For Type 1, set up the local coordinate system as shown in Fig.3. Four nodes are located on $(\bar{x}, \bar{y})$ plane in the local coordinate system, and nodes 1 and 5 are located on the $\bar{x}$ axis.

Because relative location of the nodes in two coordinate systems as shown in Figs. 1 and 3 are not changed, coordinates of additional node 9 in local coordinate system can be also written as

$$
\left\{\begin{array}{l}
\bar{x}_{9}=c_{1} \bar{x}_{4}+c_{2} \bar{x}_{5}+\left(1-c_{1}-c_{2}\right) \bar{x}_{1} \\
\bar{y}_{9}=c_{1} \bar{y}_{4}+c_{2} \bar{y}_{5}+\left(1-c_{1}-c_{2}\right) \bar{y}_{1}
\end{array}\right.
$$

Rearranging Eq.(5), while $\bar{x}_{1}, \bar{y}_{1}$ and $\bar{y}_{5}$ are all equal to 0 , we have

$$
\left\{\begin{array}{c}
\bar{x}_{9}=c_{1} \bar{x}_{4}+c_{2} \bar{x}_{5} \\
\bar{y}_{9}=c_{1} \bar{y}_{4}
\end{array}\right.
$$

Using the standard formulation of force density method, the equilibrium equations of node 9 on $(\bar{x}, \bar{y})$ plane can be written as follows:

$$
\left\{\begin{array}{c}
\left(\bar{x}_{5}-\bar{x}_{9}\right) \gamma_{u l 1}+\left(\bar{x}_{4}-\bar{x}_{9}\right) \gamma_{u l 2}-\bar{x}_{9} \gamma_{a}=0 \\
-\bar{y}_{9} \gamma_{u l 1}+\left(\bar{y}_{4}-\bar{y}_{9}\right) \gamma_{u l 2}-\bar{y}_{9} \gamma_{a}=0
\end{array}\right.
$$

where $\gamma_{a}, \gamma_{u l 1}$ and $\gamma_{u l 2}$ are the force densities of the additional strings, the first and second upper level strings, respectively.

Substituting Eq.(6) into Eq.(7), we have

$$
\left\{\begin{array}{c}
\left(\gamma_{u l 1}-c_{2} \gamma_{u l 1}-c_{2} \gamma_{u l 2}-c_{2} \gamma_{a}\right) \bar{x}_{5}+\left(-c_{1} \gamma_{u l 1}+\gamma_{u l 2}-c_{1} \gamma_{u l 2}-c_{1} \gamma_{a}\right) \bar{x}_{4}=0 \\
-c_{1} \gamma_{u l 1}+\left(1-c_{1}\right) \gamma_{u l 2}-c_{1} \gamma_{a}=0
\end{array}\right.
$$

In Eq.(8), the left-hand side of second equation is the same as coefficient of $\bar{x}_{4}$ in the first equation. So Eq.(8) can be simplified into

$$
\left\{\begin{array}{l}
\gamma_{u l 1}-c_{2} \gamma_{u l 1}-c_{2} \gamma_{u l 2}-c_{2} \gamma_{a}=0 \\
-c_{1} \gamma_{u l 1}+\left(1-c_{1}\right) \gamma_{u l 2}-c_{1} \gamma_{a}=0
\end{array}\right.
$$


From Eq.(9), $\gamma_{u l 1}$ and $\gamma_{u l 2}$ can be expressed as

$$
\begin{aligned}
& \gamma_{u l 1}=\frac{\gamma_{a} c_{1}}{1-c_{2}-c_{1}} \\
& \gamma_{u l 2}=\frac{\gamma_{a} c_{2}}{1-c_{2}-c_{1}}
\end{aligned}
$$

Using the global coordinate system, equilibrium equations of nodes 1 and 6 can be written as

$$
\left\{\begin{array}{c}
\left(x_{1}-x_{4}\right) \lambda+\left(x_{6}-x_{1}\right) \gamma_{v}+\left(x_{2}+x_{3}-2 x_{1}\right) \gamma_{b l}+\left(x_{9}-x_{1}\right) \gamma_{a}=0 \\
\left(y_{1}-y_{4}\right) \lambda+\left(y_{6}-y_{1}\right) \gamma_{v}+\left(y_{2}+y_{3}-2 y_{1}\right) \gamma_{b l}+\left(y_{9}-y_{1}\right) \gamma_{a}=0 \\
\left(z_{1}-z_{4}\right) \lambda+\left(z_{6}-z_{1}\right) \gamma_{v}+\left(z_{2}+z_{3}-2 z_{1}\right) \gamma_{b l}+\left(z_{9}-z_{1}\right) \gamma_{a}=0 \\
\left(x_{6}-x_{3}\right) \lambda+\left(x_{1}-x_{6}\right) \gamma_{v}+\left(x_{8}-x_{6}\right) \gamma_{u l 1}+\left(x_{7}-x_{6}\right) \gamma_{u l 2}=0 \\
\left(y_{6}-y_{3}\right) \lambda+\left(y_{1}-y_{6}\right) \gamma_{v}+\left(y_{8}-y_{6}\right) \gamma_{u l 1}+\left(y_{7}-y_{6}\right) \gamma_{u l 2}=0 \\
\left(z_{6}-z_{3}\right) \lambda+\left(z_{1}-z_{6}\right) \gamma_{v}+\left(z_{8}-z_{6}\right) \gamma_{u l 1}+\left(z_{7}-z_{6}\right) \gamma_{u l 2}=0
\end{array}\right.
$$

where $\lambda, \gamma_{v}$ and $\gamma_{b l}$ are the force densities of bars, declining strings and lower level strings, respectively. After substituting Eqs.(2), (3), (4) and (10) into Eq.(11) and solving it, we can obtain

$$
c_{2}=(\sqrt{3} \sin \alpha+\cos \alpha) \frac{c_{1} r_{2}}{r_{1}}-c_{1}+1
$$

Other force densities can be also obtained as

$$
\begin{aligned}
& \lambda=\frac{\gamma_{a}\left[-\sqrt{3} c_{1} r_{2} \sin (2 \alpha)+c_{1} r_{2} \sin ^{2} \alpha-\sqrt{3} c_{1} r_{1} \sin \alpha-c_{1} r_{2} \cos ^{2} \alpha+c_{1} r_{1} \cos \alpha-2 r_{1} \cos \alpha-c_{1} r_{2}\right]}{r_{1}(\sqrt{3} \sin \alpha+\cos \alpha)} \\
& \gamma_{v}=-\frac{\gamma_{a}\left(-\sqrt{3} c_{1} r_{1} \sin \alpha+4 c_{1} r_{2} \sin ^{2} \alpha+c_{1} r_{1} \cos \alpha+\sqrt{3} r_{1} \sin \alpha-r_{1} \cos \alpha-c_{1} r_{2}\right)}{r_{1}(\sqrt{3} \sin \alpha+\cos \alpha)} \\
& \gamma_{b l}=-\frac{\gamma_{a} r_{2}\left(\sqrt{3} c_{1} r_{2} \sin \alpha+c_{1} r_{2} \cos \alpha+r_{1}-c_{1} r_{1}\right)}{r_{1}^{2}(\sqrt{3} \sin \alpha+\cos \alpha)}
\end{aligned}
$$

As presented in Eq.(4), $\left(1-c_{1}-c_{2}\right)$ must be greater than 0, namely, from Eq.(12), we have

$$
(\sqrt{3} \sin \alpha+\cos \alpha) \frac{c_{1} r_{2}}{r_{1}}>0
$$

From Eq.(14), we have

$$
\tan \alpha>-\frac{\sqrt{3}}{3}
$$

Namely, $\alpha$ must be greater than $150^{\circ}$.

\subsection{Type 2}

For Type 2, we can obtain the same results as Eq.(10) through equilibrium equations at the additional node. In Type 2, equilibrium equations at nodes 1 and 6 can be written as

$$
\text { Node } 1\left\{\begin{array}{c}
\left(x_{1}-x_{4}\right) \lambda+\left(x_{6}-x_{1}\right) \gamma_{v}+\left(x_{2}+x_{3}-2 x_{1}\right) \gamma_{b l}+\left(x_{9}-x_{1}\right) \gamma_{a}=0 \\
\left(y_{1}-y_{4}\right) \lambda+\left(y_{6}-y_{1}\right) \gamma_{v}+\left(y_{2}+y_{3}-2 y_{1}\right) \gamma_{b l}+\left(y_{9}-y_{1}\right) \gamma_{a}=0 \\
\left(z_{1}-z_{4}\right) \lambda+\left(z_{6}-z_{1}\right) \gamma_{v}+\left(z_{2}+z_{3}-2 z_{1}\right) \gamma_{b l}+\left(z_{9}-z_{1}\right) \gamma_{a}=0
\end{array}\right.
$$




$$
\text { Node } 6\left\{\begin{array}{c}
\left(x_{6}-x_{3}\right) \lambda+\left(x_{1}-x_{6}\right) \gamma_{v}+\left(x_{7}-x_{6}\right) \gamma_{u l 1}+\left(x_{9}-x_{6}\right) \gamma_{u l 2}=0 \\
\left(y_{6}-y_{3}\right) \lambda+\left(y_{1}-y_{6}\right) \gamma_{v}+\left(y_{7}-y_{6}\right) \gamma_{u l 1}+\left(y_{9}-y_{6}\right) \gamma_{u l 2}=0 \\
\left(z_{6}-z_{3}\right) \lambda+\left(z_{1}-z_{6}\right) \gamma_{v}+\left(z_{7}-z_{6}\right) \gamma_{u l 1}+\left(z_{9}-z_{6}\right) \gamma_{u l 2}=0
\end{array}\right.
$$

After substituting node coordinates into Eq.(16), we have

$$
\left\{\begin{array}{c}
\left(k_{1}+2 \gamma_{b l}+2 \gamma_{a}\right) r_{1}+\gamma_{b l} r_{1}-\lambda r_{2} \cos \alpha-\gamma_{v} k_{2}-\gamma_{a}\left(c_{1} k_{2}+c_{2} r_{2} \cos \alpha-k_{3} r_{1}\right)=0 \\
-\lambda r_{2} \sin \alpha-\gamma_{v} k_{4}-\gamma_{a}\left(c_{1} k_{4}+c_{2} r_{2} \sin \alpha\right)=0 \\
-\lambda h-\gamma_{v} h-\gamma_{a}\left(c_{1}+c_{2}\right) h=0 \\
\frac{1}{k_{3}}\left[k_{5} \cos \alpha+\frac{1}{2} c_{1}^{2} \gamma_{a} r_{1}+k_{6} c_{1}-\gamma_{a} c_{2}^{2} r_{1}+\left(\gamma_{a}-\lambda+\frac{1}{2} \gamma_{v}\right) r_{1} c_{2}+\left(\lambda-\frac{1}{2} \gamma_{v}\right) r_{1}\right]=0 \\
\frac{1}{k_{3}}\left\{\left[c_{1}^{2} \gamma_{a}+\left(k_{1}-\gamma_{a} c_{2}\right) c_{1}+\gamma_{a} c_{2}^{2}+k_{1} c_{2}-\lambda-\gamma_{v}\right] r_{2} \sin \alpha-\frac{\sqrt{3}}{2} c_{1}^{2} \gamma_{a} r_{1}+k_{7}\right\}=0 \\
h\left[\left(c_{1}+c_{2}\right) \gamma_{a}+\lambda+\gamma_{v}\right]=0
\end{array}\right.
$$

where

$$
\begin{aligned}
& k_{1}=\lambda+\gamma_{v}-\gamma_{a} \\
& k_{2}=-\frac{1}{2} r_{2} \cos \alpha+\frac{\sqrt{3}}{2} r_{2} \sin \alpha \\
& k_{3}=c_{1}+c_{2}-1 \\
& k_{4}=-\frac{\sqrt{3}}{2} r_{2} \cos \alpha-\frac{1}{2} r_{2} \sin \alpha \\
& k_{5}=c_{1}^{2} \gamma_{a} r_{2}-\gamma_{a} c_{2} r_{2}-\gamma_{a} c_{2} r_{2} c_{1}+k_{1} r_{2}\left(c_{1}+c_{2}\right)+\gamma_{a} c_{2}^{2} r_{2}-\lambda r_{2}-\gamma_{v} r_{2} \\
& k_{6}=-\frac{1}{2} \gamma_{a} c_{2} r_{1}-\lambda r_{1}+\frac{1}{2} \gamma_{v} r_{1}-\frac{1}{2} \gamma_{a} r_{1} \\
& k_{7}=-\frac{\sqrt{3}}{2} c_{1} \gamma_{v} r_{1}-\frac{\sqrt{3}}{2} \gamma_{a} c_{1} c_{2} r_{1}+\frac{\sqrt{3}}{2} c_{1} r_{1} \gamma_{a}-\frac{\sqrt{3}}{2} \gamma_{v} r_{1} c_{2}+\frac{\sqrt{3}}{2} \gamma_{v} r_{1}
\end{aligned}
$$

From the sixth equation in Eq.(17), the force density of strut is written as

$$
\lambda=-c_{1} \gamma_{a}-c_{2} \gamma_{a}-\gamma_{v}
$$

After substituting Eq.(18) to the second equation of Eq.(17), we obtain

$$
\left(\gamma_{v}+\gamma_{a} c_{1}\right)\left(\sin \alpha+\sin \left(\alpha+\frac{\pi}{3}\right)\right)=0
$$

From Eq.(19), we find that either of the following equations is satisfied:

$$
\left\{\begin{array}{c}
\gamma_{v}=-c_{1} \gamma_{a} \\
\sin \alpha+\sin \left(\alpha+\frac{\pi}{3}\right)=0 \Rightarrow \alpha=150^{\circ}
\end{array}\right.
$$

Since $\gamma_{v}$ and $\gamma_{a}$ are force densities which must be positive, the first expression of Eq.(20) is not correct. Therefore $\alpha$ should be equal to $150^{\circ}$ in Type 2 .

\subsection{Type 3}

In Type 3 as shown in Fig.2(c), nodes 1, 5, 6 and 9 are located on the same plane. We can also set up equations similar to Eqs.(5)-(8), and can obtain the same results as Eq.(10). Nodes 1 and 6 are chosen to set up equilibrium equations similar to Eq.(11). Process for solving other parameters is also the same as Type 1. So analysis for Type 3 is almost the same as Type 
1 and is not described in detail here. In Type 3, we can obtain

$$
c_{1}=(\sqrt{3} \sin \alpha+\cos \alpha) \frac{c_{2} r_{2}}{r_{1}}-c_{2}+1
$$

Difference between Eqs.(12) and (21) is only that $c_{1}$ and $c_{2}$ exchange places with each other. Force densities of the members can be expressed as

$$
\begin{aligned}
& \lambda=\frac{\gamma_{a}\left[-\sqrt{3} c_{2} r_{2} \sin (2 \alpha)+c_{2} r_{2} \sin ^{2} \alpha-c_{2} r_{2} \cos ^{2} \alpha+2 c_{2} r_{1} \cos \alpha-2 r_{1} \cos \alpha-c_{2} r_{2}\right]}{r_{1}(\sqrt{3} \sin \alpha+\cos \alpha)} \\
& \gamma_{v}=-\frac{\gamma_{a}\left(4 c_{2} r_{2} \sin ^{2} \alpha+\sqrt{3} r_{1} \sin \alpha+2 c_{2} r_{1} \cos \alpha-r_{1} \cos \alpha-c_{2} r_{2}\right)}{r_{1}(\sqrt{3} \sin \alpha+\cos \alpha)} \\
& \gamma_{b l}=-\frac{\gamma_{a} r_{2}\left(\sqrt{3} c_{2} r_{2} \sin \alpha+c_{2} r_{2} \cos \alpha+r_{1}-c_{2} r_{1}\right)}{r_{1}^{2}(\sqrt{3} \sin \alpha+\cos \alpha)} \\
& \gamma_{u l 1}=\frac{\gamma_{a} c_{1}}{1-c_{2}-c_{1}} \\
& \gamma_{u l 2}=\frac{\gamma_{a} c_{2}}{1-c_{2}-c_{1}}
\end{aligned}
$$

\section{Investigation of Analytical Solutions}

\subsection{Type 1}

It is well known that the equilibrium equation with respect to the force densities is invariant with respect to affine transformation of nodal coordinates [21]. Therefore, some geometrical parameters can be assigned without loss of generality. Hence, in the following numerical examples, $H$ is assigned as $0.25 \mathrm{~m}$, and $r_{1}$ is $0.1 \mathrm{~m}$. Since the force densities of members can be simultaneously scaled, we assign $\gamma_{a}=1$. The parameter $k=r_{2} / r_{1}$ is used in the following investigation.

Based on Eq.(12), $c_{2}$ and $\left(1-c_{1}-c_{2}\right)$ are plotted with respect to $c_{1}$ and $\alpha \quad\left(\geq 150^{\circ}\right)$ in Fig. 4 , where $r_{2}=r_{1}(k=1)$, and surfaces 1 and 2 show $c_{2}$ and $\left(1-c_{1}-c_{2}\right)$, respectively. As seen in the figure, with increase of $\alpha, c_{2}$ decreases and $\left(1-c_{1}-c_{2}\right)$ increases; with increase of $c_{1}, c_{2}$ decreases and $\left(1-c_{1}-c_{2}\right)$ increases. When $c_{1}$ is equal to $0, c_{2}$ is always equal to 1 and $\left(1-c_{1}-c_{2}\right)$ is always equal to 0 ; namely the first and second upper level strings are on the same lines, which means that the added nodes 7,8 and 9 cannot be at equilibrium and the structures are not self-equilibrated. Furthermore, if $\alpha$ is equal to $150^{\circ}$, then $\left(1-c_{1}-c_{2}\right)$ is equal to 0 . When $\alpha$ is greater than $150^{\circ},\left(1-c_{1}-c_{2}\right)$ is always greater than 0 . So $\alpha$ should be greater than $150^{\circ}$, which is the same as conclusion obtained from Eq.(15).

It is seen from Fig. 4 that the range of $c_{1}$ decreases with increase of $\alpha$. When $c_{2}$ is equal to $0, c_{1}$ reaches the following maximal value $c_{1}^{\max }$ as a function of $\alpha$ and $k$ :

$$
c_{1}^{\max }=\frac{1}{1-k(\sqrt{3} \sin \alpha+\cos \alpha)}
$$

Therefore, the range of $c_{1}$ is found as $c_{1} \in\left(0,[1-k(\sqrt{3} \sin \alpha+\cos \alpha)]^{-1}\right)$. It is also observed from Fig.4 that the maximum value of $c_{1}$ is always greater than or equal to 0.5. Moreover, the greater $c_{1}$ is, the more obvious influence $\alpha$ has on $c_{2}$. 
The relation in Fig.4 also depends on the parameter $k=r_{2} / r_{1}$. So assign $c_{1}$ as 0.4 here to investigate influence of $\alpha$ and $k$ on $c_{2}$. Curved surfaces 1 and 2 in Fig.5 are the plots of $c_{2}$ and $c_{1}^{\max }$, respectively, with respect to $k$ and $\alpha$. As seen from surfaces 1 and 2, with increase of $k$, both of $c_{2}$ and $c_{1}^{\max }$ decrease. With increase of $\alpha$, the two values also decreases.

As discussed by Zhang and Ohsaki [16], there are always at least four zero eigenvalues in the force density matrix of the self-equilibrated structures. This condition is called self-equilibrium conditions here. Substituting 0.1 to $r_{1}$ and $r_{2}(k=1)$ in Eq. (12), we obtain

$$
c_{2}=c_{1}(\cos \alpha+\sqrt{3} \sin \alpha)-c_{1}+1
$$

Then suppose $c_{1}$ can vary in the range $\left(0,(1-\sqrt{3} \sin \alpha-\cos \alpha)^{-1}\right)$, and obtain some groups of node coordinates in Type 1 .

Figure 6 shows the structures in Type 1 when $\alpha>150^{\circ}$. It is seen from Eq.(24) that $c_{2}$ is a linear function of $c_{1}$ when $\alpha$ is given a priori. With variation of $c_{1}$ in the range $\left(0,(1-\sqrt{3} \sin \alpha-\cos \alpha)^{-1}\right)$, each additional node moves along a line, as shown in Fig. 6 , on the triangle that is defined by three existing nodes and enclose the additional node. We confirmed that the self-equilibrium conditions in reference [16] are always satisfied. When value of $c_{1}$ is not in its range, the additional nodes will move outside of the triangles, and internal forces of the members connected to the additional nodes cannot keep the nodes at equilibrium, so the structure is not self-equilibrated. Moreover, there will appear negative eigenvalues of the force density matrix and the structure is not super-stable.

Figure 7 shows a physical model of Type 1. It is self-equilibrated with $\alpha=175^{\circ}$. As seen in Fig. 7(b), the bars are close to each other, but they do not physically contact; however, because the bars and additional strings are all located inside of the cylinder, interferences among the members easily occur.

An example with $\alpha<150^{\circ}$ of Type 1 is shown in Fig.8. Although the additional nodes are still located on the corresponding lines, they have moved out of the triangles which enclose them. Three strings connected at every additional node can't keep the additional node at equilibrium. Therefore, $\alpha$ must be greater than $150^{\circ}$ so that the structure is at self-equilibrium. This fact is similar to conclusion from Eq.(15).

\subsection{Type 2}

As presented in Section 4.2, $\alpha$ should be equal to $150^{\circ}$ in Type 2. We investigate six equations in Eq.(17), which are the equilibrium equations of nodes 1 and 6. Since $\gamma_{b l}$ exists only in the first equation of Eq.(17), it can be solved to obtain $\gamma_{b l}$. From the sixth equation in Eq.(17), the force density of bar can be expressed as Eq.(18). It is difficult to derive expressions of $c_{1}$ and $c_{2}$ with symbolic form of $\gamma_{a}$, and the expressions can be obtained only when $\gamma_{a}$ is given. For example, when $\gamma_{a}$ is equal to 1.4, after substituting Eq.(18) and 
$\alpha=150^{\circ}$ to the fifth equation of Eq.(17) and solving it, we can obtain

$$
\gamma_{v}=\frac{c_{1}\left(1.4 c_{1}+3.825 c_{2}-1.4\right)}{1-c_{1}-c_{2}}
$$

After substituting Eq.(25) into the fourth equation of Eq.(17), $c_{2}$ can be solved as

$$
c_{2}=\frac{c_{1}\left(0.596 c_{1}-1.809\right)}{1-1.057 c_{1}}
$$

The second and third equations of Eq.(17) are linearly related to the sixth equation of Eq.(17); so all force densities and nodal locations are determined by assigning $\gamma_{a}$ and $c_{1}$.

So relation between $c_{1}$ and $c_{2}$ depends on $\gamma_{a}$, and different $\gamma_{a}$ results in different location of the additional node. When an arbitrary positive force density is assigned to the additional strings, the additional nodes are always located in the triangles which enclose them; namely, only if the additional strings are in the triangles which enclose them, the structure can satisfy the self-equilibrium condition and is at self-equilibrium.

\subsection{Type 3}

Because Eq.(21) can be obtained by exchanging $c_{1}$ and $c_{2}$ in Eq.(12), conclusions from Eq.(12) can be also applicable to Type 3; i.e., $\alpha$ should be greater than $150^{\circ} ; c_{1}$ is a decreasing function of both $\alpha$ and $c_{2}$; and $c_{2}$ reaches the following maximal value $c_{2}^{\max }$ when $c_{1}$ is equal to 0 :

$$
c_{2}^{\max }=\frac{1}{1-k(\sqrt{3} \sin \alpha+\cos \alpha)}
$$

Hence, $c_{2} \in\left(0,[1-k(\sqrt{3} \sin \alpha+\cos \alpha)]^{-1}\right)$. In the similar manner as Section 5.1, $c_{2}^{\max }$ is an decreasing function of $k$ and $\alpha$.

Figure 9 shows an example of geometry realization of the Type 3 structure. As seen in Fig.9, each additional node moves along a line as $c_{2}$ is varied; namely, as long as the additional nodes are on the lines within the corresponding triangles, the self-equilibrium condition is always satisfied. When $c_{2}$ is outside of its range, the additional nodes move out of the triangles and equilibrium conditions are not satisfied with tensile forces in strings; accordingly, there are negative eigenvalues of the force density matrix and the structure is not stable. Figure 10 shows the Type 3 structure with $\alpha=170^{\circ}$, which confirms that the Type 3 structure can be self-equilibrated when $\alpha$ is greater than $150^{\circ}$.

When $\alpha<150^{\circ}$, the additional nodes move out of the triangles which enclose them, as shown in Fig.11; hence, the structure in Fig.11 is not at self-equilibrium. So in Type 3, $\alpha$ should be greater than $150^{\circ}$. 


\section{Numerical Investigation of Internal Forces}

Detailed investigations of internal forces are given in this section for Type 1 and 3 structures. Type 2 is excluded, because it allows only limited shape with $\alpha=150^{\circ}$.

\subsection{Type 1}

Based on the expressions (10) and (13) for force densities of Type 1 structure, we will analyze relations between internal forces of the members and other parameters. In Figs. 12-15, the vertical axis refers to ratio of internal forces of each member to the force of additional string. We found in Figs. 4 and 5 in Section 5 that the maximum value of $c_{1}$ is always greater than 0.5 . Moreover, when $c_{1}$ is equal to its maximal value, $c_{2}$ is equal to 0 ; when $c_{1}$ is equal to $0, c_{2}$ is equal to 1 . So we assign an intermediate value 0.3 to $c_{1}$. Figure 12 shows the values of internal forces with respect to $\alpha$. Here let $f_{\mathrm{b}}, f_{\mathrm{v}}, f_{\mathrm{b} 1}, f_{\mathrm{a}}, f_{\mathrm{ul} 1}$ and $f_{\mathrm{ul} 2}$ represent internal forces of the bars, declining strings, lower level strings, additional strings, the first and second upper level strings, respectively.

It is seen from Fig. 12 that, with increase of $\alpha, f_{\mathrm{a}}$ is almost constant and absolute values of other internal forces decrease. Distribution of the internal force is uneven as $\alpha$ is close to $150^{\circ}$. When $\alpha$ is equal to $150^{\circ}, f_{\mathrm{v}}$ and $f_{\mathrm{ul} 2}$ tend to infinity. So $\alpha$ cannot be equal to $150^{\circ}$.

When $\alpha$ is equal to $180^{\circ}$, ratio of the maximal force to the minimal force of string reaches 50 and distribution of the internal force is also uneven.

When $\alpha$ is about $165^{\circ}$, distribution of the internal force is most even. So we set $\alpha=165^{\circ}$ to analyze influence of $c_{1}$ on internal forces. Figure 13 shows variations of internal forces of the members with respect to $c_{1}$. As seen in Fig. 13, with increase of $c_{1}, f_{\text {ull }}$ increases, $f_{\mathrm{a}}$ is almost constant and absolute values of other forces all decrease.

In Type 1 , node 9 connected to node 1 is supported by the two upper level strings connecting node 9 to nodes 4 and 5. With increase of $c_{1}$, node 9 moves from node 4 to 5 . When $c_{1}$ becomes close to 0 , namely, node 9 approaches to node 4 , there is apparent uneven distribution of internal forces. When $c_{1}$ approaches its maximal value, node 9 moves toward node 5 , and $f_{\mathrm{bl}}, f_{\mathrm{v}}$ and $f_{\mathrm{ul} 2}$ are all equal to 0 resulting in uneven distribution of internal forces. So when additional nodes are not close to any existing node, the internal forces are distributed more evenly.

\subsection{Type 3}

Using expressions (22) of force densities in Type 3, influences of other parameters on internal forces are investigated. Figure 14 shows variations of internal forces with respect to $c_{2}$ in Type 3 when $\alpha=165^{\circ}$. As seen in Fig. 14, with increasing of $c_{2}, f_{\mathrm{ul} 2}$ increases, $f_{\mathrm{a}}$ is almost constant, and absolute values of other forces all decrease. In Type 3, node 9 connected to node 1 is located to the first and second upper level strings connecting node 9 to nodes 5 and 6 , respectively. When $c_{1}$ increases, node 9 moves toward node 5. Conversely, node 9 approaches to node 6 . When node 9 becomes close to node 6 , i.e, $c_{1}=1, f_{\text {ull }}$ will increase rapidly to 52.23 , while 
absolute values of all other forces are below 5. When $c_{2}$ becomes larger and node 9 approaches to node $5, f_{\mathrm{b}}, f_{\mathrm{bl}}$ and $f_{\mathrm{ul} 1}$ decrease to 0 . So when additional nodes approach the existing nodes, uneven distribution of the internal forces becomes apparent. This is the same as Type 1 . Moreover, when $c_{2}$ is beyond $0.53, f_{\mathrm{v}}$ becomes negative and equilibrium conditions are not satisfied with positive forces in strings; i.e., the structure is not stable.

Internal forces when $c_{1}=0.3$ are plotted with respect to $\alpha$ in Fig. 15 , which shows that with increase of $\alpha, f_{\mathrm{a}}$ is almost constant and absolute values of all other force densities decrease. When $\alpha$ approaches to $150^{\circ}$ or $180^{\circ}$, difference among the internal forces becomes large. This is the same as observed in Type 1 .

Through the above numerical analysis, we can obtain the following conclusions for Types 1 and 3 structures:

1) Distribution of the internal forces becomes uneven as the additional nodes approach to existing nodes.

2) When $\alpha$ approaches to $150^{\circ}$ or $180^{\circ}$, there are apparent uneven distribution of the internal forces.

When $\alpha=150^{\circ}$, the 3-bar prismatic tensegrity unit without additional strings is self-equilibrated. For Types 1 and 3, distribution of internal forces and the value of $\alpha$ change as the forces of the additional strings are increased. Moreover, the structure is self-equilibrated if additional nodes are in the triangles which enclose them. If we allow uneven distribution of internal forces, the range of $\alpha$ should be $\left[150^{\circ}, 180^{\circ}\right]$ in Types 1 and 3.

In Type 2, it is difficult to obtain general expressions of force densities, because relation between $c_{1}$ and $c_{2}$ depends on $\gamma_{a}$. Relations between internal forces of the members and other parameters are difficult to be investigated.

Because it has been presented that the two 3-bar tensegrity units when $\alpha=150^{\circ}$ and $-150^{\circ}$ are symmetrical, we can deduce easily characteristics of new configurations when $\alpha=-150^{\circ}$ from conclusions of $\alpha=150^{\circ}$. So analysis for new configurations when $\alpha=-150^{\circ}$ is not described here.

\section{Conclusions}

In the paper, we presented three new types of tensegrity units by adding strings and nodes to the well-known 3-bar prismatic tensegrity unit. The strings are added between the nodes on the lower plane and the level strings on the upper plane. The three types are characterized by the connectivity of the additional string to the additional nodes located on the upper level strings.

Type 1 and 3 structures have the following characteristics: 
(1) Additional node 9 connected to node 1 should be located between nodes 4 and 5 or 5 and 6. The tensegrity units when $\alpha>0$ are always self-equilibrated when $\alpha \in\left[150^{\circ}, 180^{\circ}\right]$. The tensegrity units when $\alpha<0$ are always at self-equilibrium when $\alpha \in\left[-180^{\circ},-150^{\circ}\right]$.

(2) The additional nodes are located on the lines which are obtained through analysis in Sections 4 and 5. Moreover they must stay in the triangles which enclose them.

(3) As the additional nodes approach to existing nodes, distribution of the internal forces becomes uneven; conversely, distribution of the internal forces becomes uniform if the additional node is sufficiently apart from the existing nodes.

(4) When $\alpha$ approaches to $\pm 150^{\circ}$ or $\pm 180^{\circ}$, there are apparent uneven distribution of the internal forces.

Type 2 structure can maintain self-equilibrated only when $\alpha= \pm 150^{\circ}$. Since the equilibrium shape is strongly limited, we did not investigated details of Type 2 structure.

\section{Acknowledgment}

The authors gratefully acknowledge the contribution of National Natural Science Fund of China (Grant Number 51605111, 51675114) and JSPS KAKENHI (Grant Number 15KT0109).

\section{Reference}

[1] R. Motro, L. Jia and B. Yang. Tensegrity-From art to structure engineering. Building Structures 2011;12:12-19.

[2] J. Cai and J. Feng. From-finding of tensegrity structures using an optimization method. Engineering Structures, 2015; 104:26-132.

[3] C. Sultan. Tensegrity: 60 years of art, science, and engineering. Advances in applied mechanics 2009;43:69-145.

[4] A. Agogino, V. SunSpiral and D. Atkinson. Super Ball Bot-Structures for Planetary Landing and Exploration. NASA Innovative Advanced Concepts (NIAC) Program 2013(7).

[5] A. Amendola, E.H. Nava, R. Goodall, I. Todd, R.E. Skelton and F. Fraternali. On the additive manufacturing, post-tensioning and testing of bi-material tensegrity structures. Composite Structures 2015;131:66-71.

[6] N. Ashwear, G. Tamadapu and A. Eriksson. Optimization of modular tensegrity structures for high stiffness and frequency separation requirements. International Journal of Solids and Structures, 2016; 80:297-309.

[7] Y. Koizumi, M. Shibata and S. Hirai. Rolling tensegrity driven by pneumatic soft actuators. In: 2012 IEEE International Conference on Robotics and Automation, RiverCentre, Minnesota, USA; 2012:1988-1993.

[8] W. Du, S. Ma, B. Li, M. Wang and S. Hirai. Dynamic simulation for 6-strut tensegrity robots. In: Proceeding of the IEEE International Conference on Information and Automation, Hailar, China;2014: 870-875. 
[9] S. Hirai and R. Imuta. Dynamic simulation of six-strut tensegrity robot rolling. In: Proceedings of the 2012 IEEE International Conference on Robotics and Biomimetics, Guangzhou, China;2012: 198-204.

[10] K. Caluwaerts and J.P. Carbajal. Energy conserving constant shape optimization of tensegrity structures. International Journal of Solids and Structures 2015;58:117-127.

[11] H. Liu, J. Geng and A. Luo. Tensegrity configuration method on connecting tensegrity units along their axes. Composite Structure 2017;162:341-350.

[12] A. Luo, Q. Li, H. Liu, J. Cheng and L, Xu. Establishment and mechanical properties analysis of spherical tensegrity structures. In: 5th Structural Engineers World Congress(SEWC 2015), Singapore; 2015:19-22.

[13] S. Pellegrino. Structural computations with the singular value decomposition of the equilibrium matrix. International Journal of Solids and Structures 1993;30(21): 3025-3035.

[14] P. Zhang and J. Feng. Initial prestress design and optimization of tensegrity systems based on symmetry and stiffness. International Journal of Solid and Structures 2017;106:68-90.

[15] X. Xu, S. Li and Y. Luo. Form-finding of a new kind of tensegrity tori using overlapping modules. Mechanics Research Communications 2017;84:1-7.

[16] J. Y. Zhang and M. Ohsaki. Tensegrity Structures: Form, Stability, and Symmetry. Springer;2015.

[17] A.G. Tibert and S. Pellegrino. Review of form-finding methods for tensegrity structures. International Journal of Space Structures 1986;26(3):241-255.

[18] M. Schenk, J.L. Herder and S.D. Guest. Design of a statically balanced tensegrity mechanism. In: ASME 2006 International Design Engineering Technical Conferences \& Computers and Information in Engineering Conference, Pennsylvania, USA; 2006:10-13.

[19] P. Zhang, K. Kawaguchi and J. Feng. Prismatic tensegrity structures with additional cables: Integral symmetric states of self-stress and cable-controlled reconfiguration procedure. International Journal of Solids and Structures 2014;51:4294-4306.

[20] Maplesoft, Maple 2016 User Manual, 2016.

[21] J. Y. Zhang and M. Ohsaki. Stability conditions for tensegrity structures. International Journal of Solids and Structures 2007;44(11-12):3875-3886. 


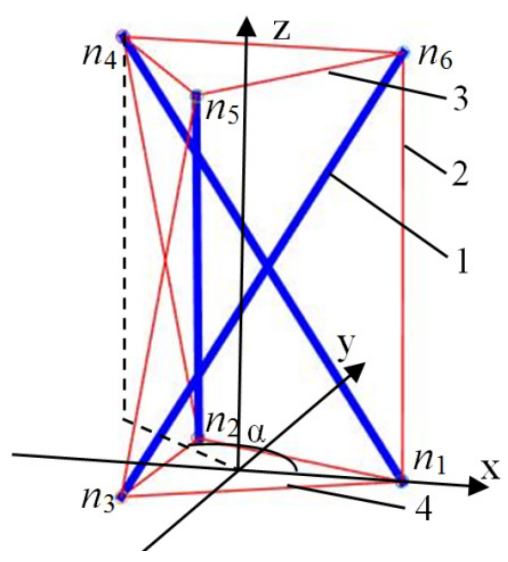

(a)

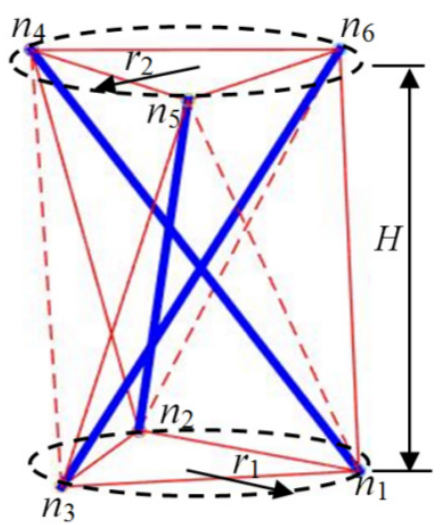

(b)

Fig. 1 3-bar prismatic tensegrity unit and its configuration; 1: bar, 2: declining string, 3: upper level string, 4: lower level string

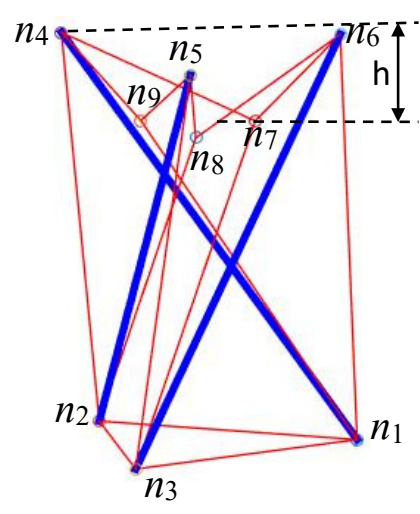

(a) Type 1

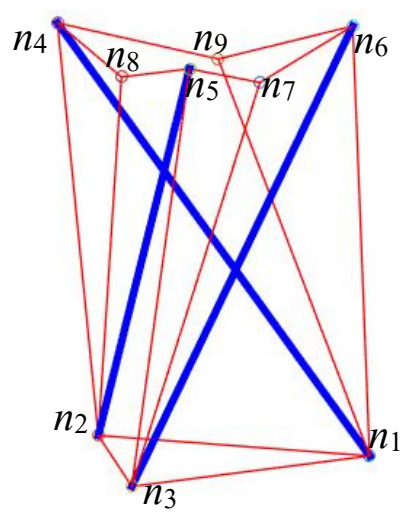

(b)Type 2

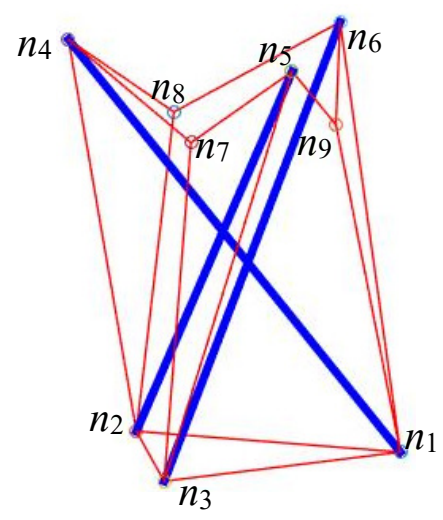

(c) Type 3

Fig.2 Three types of 3-bar prismatic tensegrity units with the additional nodes and strings

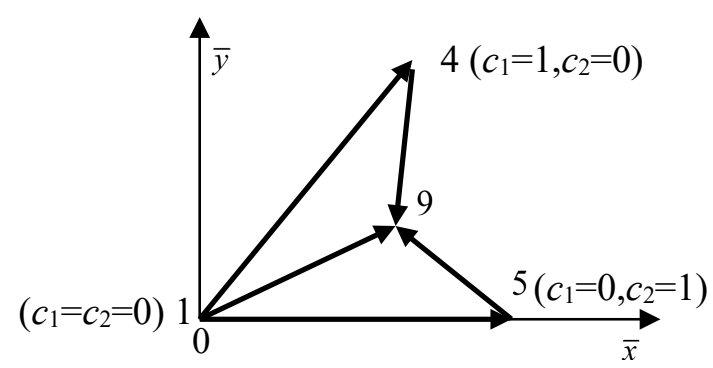

Fig. 3 Nodes 1, 4, 5 and 9 in local coordinate system 


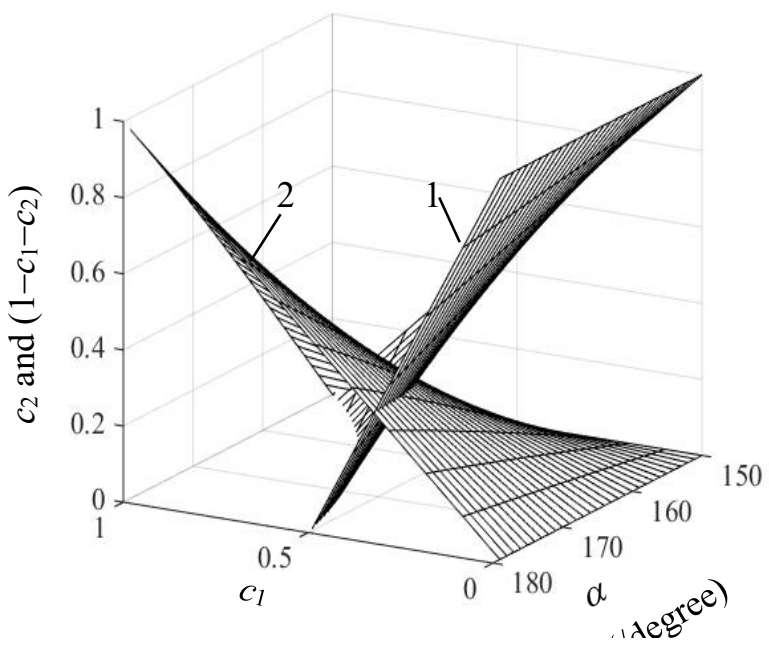

Fig.4 Variations of $c_{2}$ and $\left(1-c_{1}-c_{2}\right)$ with respect to $c_{1}$ and $\alpha ; 1: c_{2}, 2:\left(1-c_{1}-c_{2}\right)$

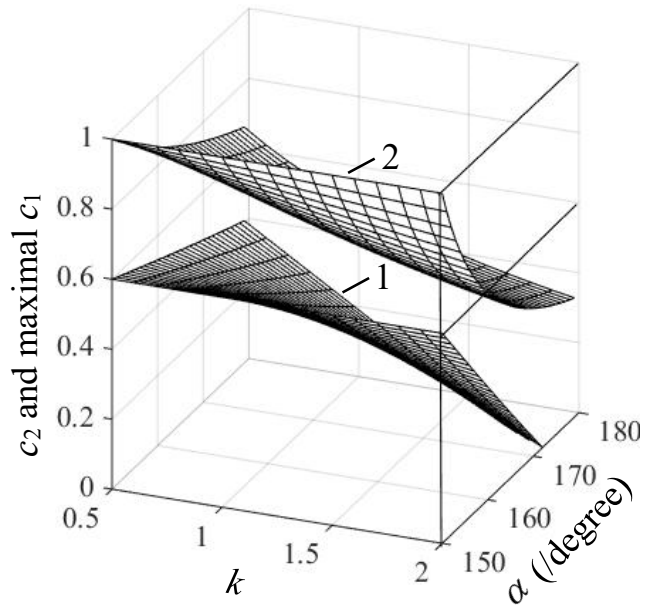

Fig.5 Variations of $c_{2}$ and $c_{1}^{\max }$ with respect to $k$ and $\alpha ; 1: c_{2}, 2: c_{1}^{\max }$

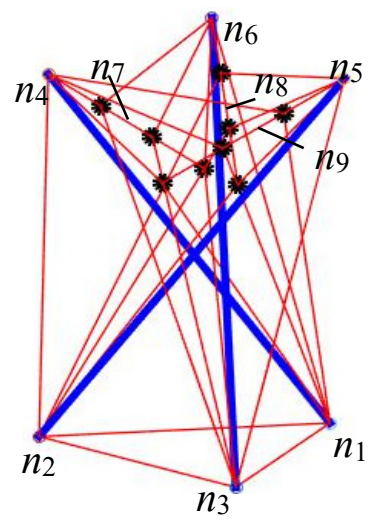

Fig.6 Analysis results in Type1 when $\alpha>150^{\circ}$ 


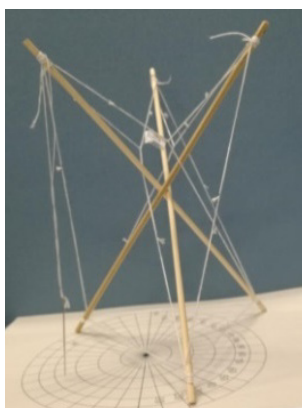

(a) Model

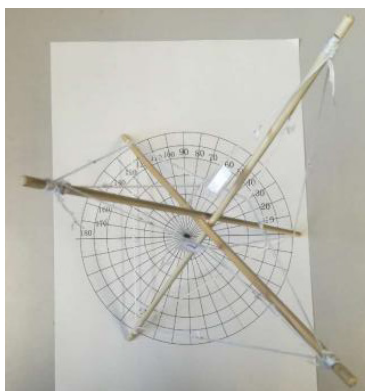

(b) Top view

Fig.7 A self-equilibrated configuration of Type 1

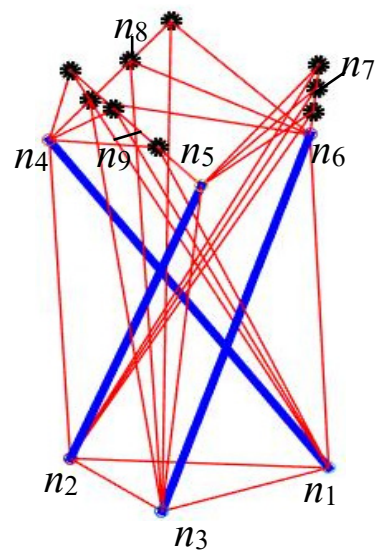

Fig. 8 Analysis results of Type 1 when $\alpha<150^{\circ}$

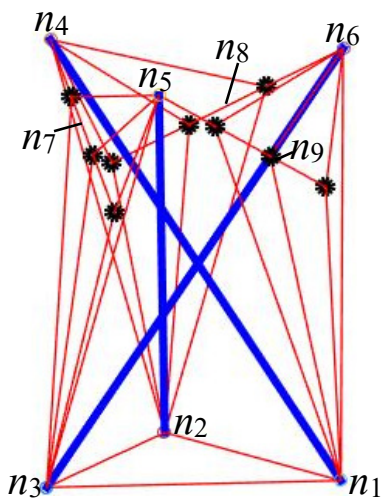

Fig.9 An example structure of Type 3 with $\alpha \geq 150^{\circ}$

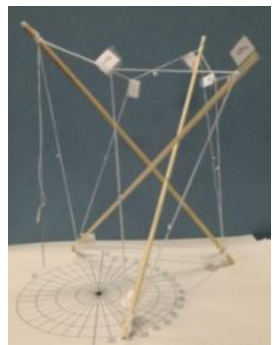

(a) Model

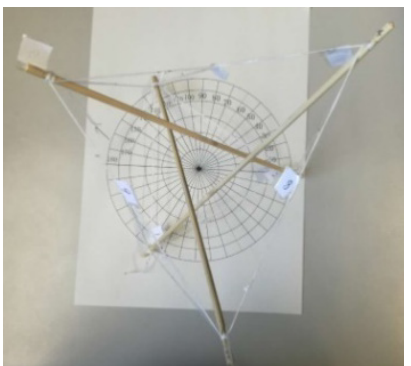

(b)Top view

Fig.10 A Physical model of Type 3 


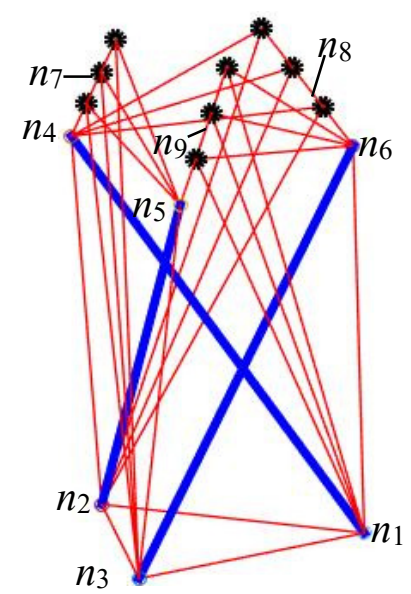

Fig.11 Analysis results in Type 3 when $\alpha<150^{\circ}$

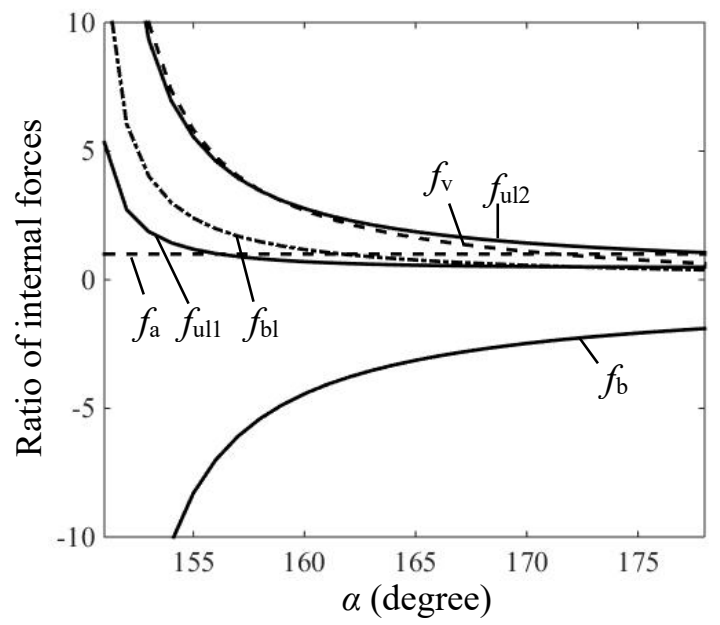

Fig.12 Variations of internal forces with respect to $\alpha$ of Type 1 structure with $c_{1}=0.3$

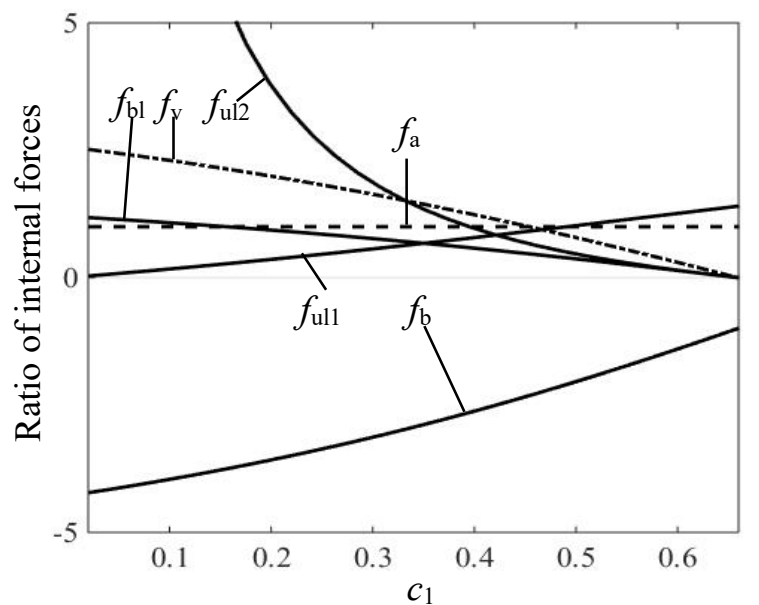

Fig. 13 Variations of ratio of internal forces with respect to $c_{1}$ of Type 1 structure with $\alpha=165^{\circ}$ 


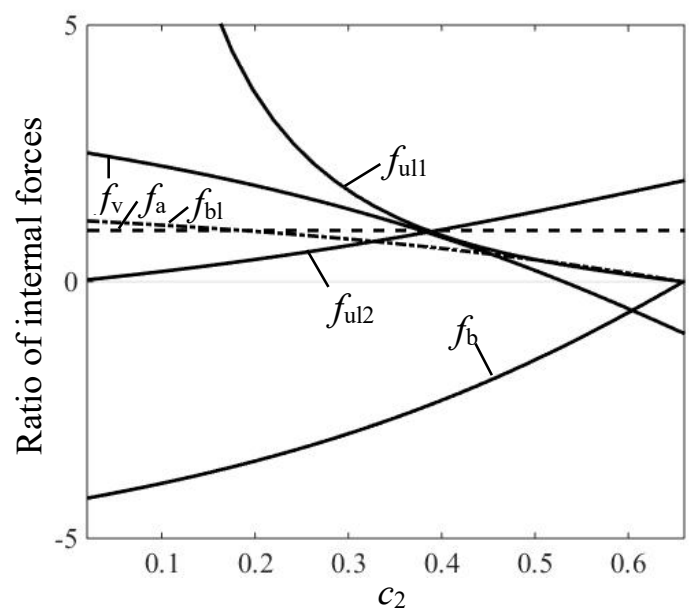

Fig.14 Variations of ratio of internal forces with respect to $c_{2}$ of Type 3 structure with $\alpha=165^{\circ}$

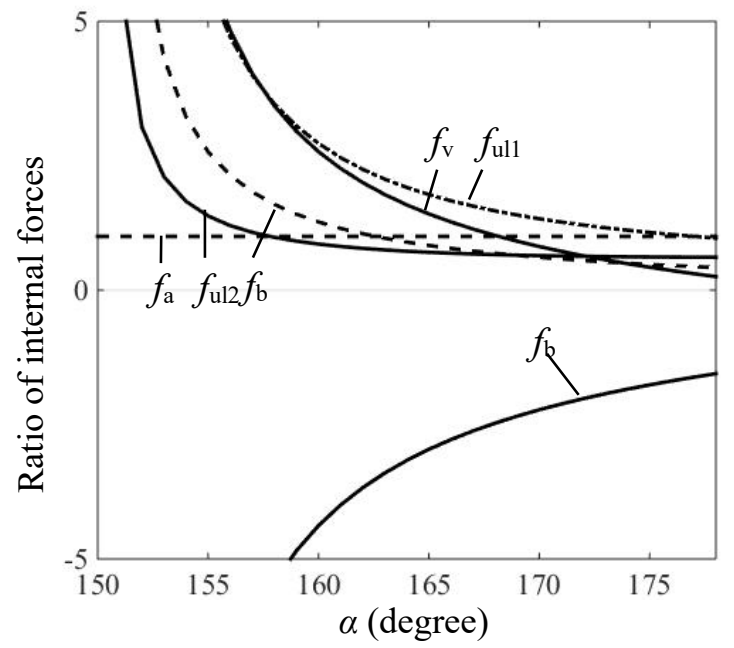

Fig. 15 Curves of ratio of internal forces with respect to $\alpha$ in Type 3 with $c_{2}=0.3$

Table 1 Node groups of Types 1,2 and 3

\begin{tabular}{c|ccc}
\hline Type & \multicolumn{3}{|c}{ Node group } \\
\hline Type 1 in Fig.2(a) & $\{1,9,4,5\}$, & $\{3,7,6,4\}$, & $\{2,8,5,6\}$ \\
\hline Type 2 in Fig.2(b) & $\{1,9,6,4\}$, & $\{3,7,5,6\}$, & $\{2,8,4,5\}$ \\
\hline Type 3 in Fig.2(c) & $\{1,9,5,6\}$, & $\{3,7,4,5\}$, & $\{2,8,6,4\}$ \\
\hline
\end{tabular}

